\title{
The Role of Nrf2 in Liver Disease: Novel Molecular Mechanisms and Therapeutic Approaches
}

\section{OPEN ACCESS}

Edited by:

Águeda González Rodríguez, Instituto de Investigación Sanitaria del Hospital Universitario de La Princesa,

Spain

Reviewed by:

Luigi Brunetti,

Università degli Studi G. d'Annunzio

Chieti e Pescara, Italy

Pallavi R. Devchand,

University of Calgary, Canada

*Correspondence:

Qiang Xia

xiaqiang@shsmu.edu.cn

Xiaoni Kong

xiaoni-kong@126.com

${ }^{\dagger}$ These authors shared co-first authorship

Specialty section:

This article was submitted to Inflammation Pharmacology,

a section of the journal

Frontiers in Pharmacology

Received: 23 August 2018

Accepted: 19 November 2018

Published: 08 January 2019

Citation:

Xu D, Xu M, Jeong S, Qian Y, Wu H, Xia Q and Kong X (2019) The Role of Nif2 in Liver Disease: Novel Molecular Mechanisms

and Therapeutic Approaches.

Front. Pharmacol. 9:1428

doi: 10.3389/fphar.2018.01428

\author{
Dongwei $\mathrm{Xu}^{1 \dagger}$, Min $\mathrm{Xu}^{1+}$, Seogsong Jeong ${ }^{1}$, Yihan Qian' ${ }^{2}$, Hailong $W_{u^{3}}$, Qiang $\mathrm{Xia}^{1 *}$ and \\ Xiaoni Kong ${ }^{1 *}$
}

'Department of Liver Surgery, Renii Hospital, School of Medicine, Shanghai Jiao Tong University, Shanghai, China, ${ }^{2}$ School of Pharmacy, Fudan University, Shanghai, China, ${ }^{3}$ Shanghai Key Laboratory for Molecular Imaging, Collaborative Research Center, Shanghai University of Medicine and Health Sciences, Shanghai, China

Oxidative stress and inflammation are the most important pathogenic events in the development and progression of liver diseases. Nuclear erythroid 2-related factor 2 (Nrf2) is the master regulator of the cellular protection via induction of anti-inflammatory, antioxidant, and cyto-protective genes expression. Multiple studies have shown that activation or suppression of this transcriptional factor significantly affect progression of liver diseases. Comprehensive understanding the roles of Nrf2 activation/expression and the outcomes of its activators/inhibitors are indispensable for defining the mechanisms and therapeutic strategies against liver diseases. In this current review, we discussed recent advances in the function and principal mechanisms by regulating Nrf2 in liver diseases, including acute liver failure, hepatic ischemia-reperfusion injury (IRI), alcoholic liver disease (ALD), viral hepatitis, non-alcoholic fatty liver disease (NAFLD), non-alcoholic steatohepatitis (NASH), and hepatocellular carcinoma (HCC).

Keywords: Nrf2, oxidative stress, cytoprotective genes, acute liver injury, viral hepatitis, non-alcoholic fatty liver disease, non-alcoholic steatohepatitis, hepatocellular carcinoma

\section{INTRODUCTION}

Oxidative stress and inflammation are the most important pathogenic events in liver diseases. During liver injuries, the unregulated production of free radicals and/or ROS leads to damage of important biomolecules and cells and generation of proinflammatory genes. Antioxidant and anti-inflammatory therapy has been considered to be beneficial in liver diseases. Nrf2 is the master regulator of the primary means of cellular defense through mediation of antioxidant response, antiinflammatory and cytoprotective properties, and dysregulation of Nrf2 activity has been revealed to correlated with the development of chronic inflammatory diseases (Alam et al., 1999; Vomund et al., 2017; Bellezza et al., 2018; Hennig et al., 2018). The protective effects of Nrf2 signaling pathway has been identified in a number of disease models, including acute kidney, lung or neurons

Abbreviations: ALD, alcoholic liver disease; ALP, alkaline phosphatase; ALT, alanine aminotransferase; AST, aspartate aminotransferase; CDDO-Im, CDDO-imidazolide; CYP2E1, cytochrome P450 2E1; D3T, 3H-1,2 dithiole-3-thione; D-GalN, D-galactosamine; DAMP, damage-associated molecular pattern; DILI, drug-induced liver injury; FGF19, fibroblast growth factor 19; GST, glutathione S-transferase; HBV, hepatitis B virus; HBx, HBV stimulates by its X protein; HCC, hepatocellular carcinoma; HCV, hepatitis C virus; IRI, ischemia-reperfusion injury; JNK, c-Jun N-terminal kinase; Keap1, Kelch-like ECHassociated protein; LDH, lactate dehydrogenase; LPS, lipopolysaccharide; NAFLD, non-alcoholic fatty liver disease; NASH, non-alcoholic steatohepatitis; NQO1, NAD(P)H quinone dehydrogenase one; Nrf2, nuclear erythroid 2-related factor 2; NS, non-structural; PBC, primary biliary cholangitis; $\operatorname{PPAR} \gamma$, peroxisome proliferator-activated receptor- $\gamma$; ROS, reactive oxygen species; VLDLR, very-low density lipoprotein receptor. 
injury, emphysema, and sepsis (Thimmulappa et al., 2007; Reddy et al., 2009; Sussan et al., 2009; Zhang et al., 2012; Liu et al., 2014). Accumulating evidence also has implicated this transcription factor in various liver diseases, including acute hepatoxicity, NAFLD, NASH, ALD, DILI, viral hepatitis, liver fibrosis, hepatic IRI, and primary hepatic malignancies (Klaassen and Reisman, 2010; Tang et al., 2014). Under acute and chronic oxidative stress and inflammatory conditions, Nrf2 is activated and prevents oxidative and inflammatory diseases by modulating genes expression of cytoprotective proteins and enzymes, which decreases ROS levels, inflammation, and cell death (Bataille and Manautou, 2012). However, the function of Nrf2 is not always protective in diseases, recent studies have identified that the gene expression of Nrf2 was associated with the pathogenesis, progression, and metastasis of cancer, resistance to cancer therapy, and the regulation of cancer cells metabolism, thereby suggesting that Nrf2 is a pleiotropic transcriptional factor (Karin and Dhar, 2016; Rojo de la Vega et al., 2018). In this review, we summarized up-to-date studies in the understanding of the roles and mechanisms of Nrf2 and the therapeutic approaches by targeting Nrf2 in liver diseases.

\section{ACTIVATION OF Nrf2 ATTENUATES ACUTE LIVER INJURY}

Study has shown that activation of Nrf2 attenuates acute liver injury. Wu et al. (2012) compared serum ALT, LDH, hepatic hemorrhage, and necrosis levels between Nrf2-null and Nrf2-enhanced mice in cadmium-induced acute liver injury mice model; they found that Nrf2-enhanced mice were associated with lower ALT and LDH levels and with fewer morphological alterations. The mRNA levels of cytoprotective genes, including sulfiredoxin-1, glutamate-cysteine ligase, and glutathione peroxidase- 2 were expressed only in Nrf2-enhanced mice, suggesting that Nrf2 activation prevents oxidative stress and acute liver injury through modulation of antioxidant defense-associated genes (Figure 1). Subsequently, the protective effects of Nrf2 was tested in LPS and D-GalN-induced liver injury mouse models by treatment with mangiferin, which could upregulate the gene expression of Nrf2 in a dose-dependent manner (Pan et al., 2016). Mangiferin treatment suppressed serum levels of ALT, AST, IL- $1 \beta$, TNF- $\alpha$, and ROS levels, adding evidences that activation of $\mathrm{Nrf} 2$ pathway protects against acute liver injury. Biochanin A, morin, curcumin, andrographolide, oxymatrine, and madecassoside were also found to play a protective role via activation of Nrf2 in LPS and D-GalNinduced acute liver injury in mice (Liu et al., 2016; Pan et al., 2017; Tian et al., 2017; Xie et al., 2017; Wang et al., 2018). In addition, the antioxidant pathway of $\mathrm{Nrf} 2$ was further tested and found to be effective in carbon tetrachloride-induced and acetaminophen-induced mouse acute liver injury models (Huang et al., 2016; Cao et al., 2017; Peng et al., 2018; Shen Z. et al., 2018). The role of Nrf2 in hepatic IRI was also identified by several studies (Ke et al., 2013; Kudoh et al., 2014; Rao et al., 2015; Ge et al., 2017; Xu et al., 2017). Ke et al. (2013) showed that the Keap1-Nrf2 complex could alleviate oxidative injury in

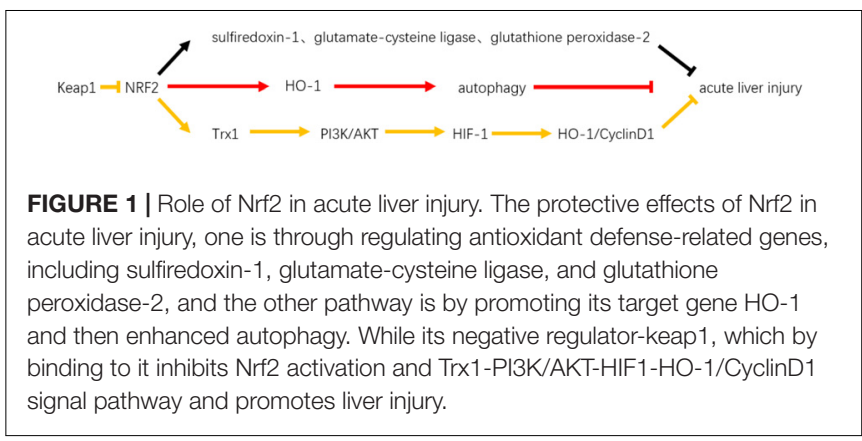

mouse orthotopic liver transplantation through Keap1 signaling (Figure 1). The protective effects were identified by limiting hepatic inflammatory responses and hepatocellular necrosis. Recently, our research identified cytoprotective effects of CDDOIm, a potent activator of the Nrf2 pathway, in hepatic IRI, through inducing Nrf2 target gene HO-1 expression leads to enhanced autophagy in hepatocytes, which results in increased clearance of damaged mitochondria, reduced mtDNA release and ROS production leading to reductions in DAMP release-induced inflammatory responses and subsequent secondary hepatocyte injury (Xu et al., 2017). Despite accumulating evidences, Nrf2based treatment is yet to enter clinical trials in the $\mathrm{USA}^{1}$ for patients with acute liver failure.

\section{ACTIVATION OF Nrf2 AMELIORATES ALCOHOLIC LIVER DISEASE}

Alcohol consumption has been revealed to be significantly associated with the development and progression of liver diseases over decades (Shepard et al., 2010). Alcohol metabolism in the liver includes ethanol oxidation by alcohol dehydrogenase in hepatocytes and microsomal oxidation promoted by CYP2E1 (Bae et al., 2011; Wang et al., 2014a). Alcohol dehydrogenaseassociated ethanol metabolism results in acetaldehyde, which gives rise to some downstream effects, such as depletion of glutathione, lipid peroxidation, and generation of ROS (Dey and Cederbaum, 2006). In addition, the dysregulation of antioxidant glutathione by Nrf2-dependent regulation was found to contribute to the development of ALD by providing pathological conditions, whereas the Nrf2-mediated antioxidant response provided protection against alcohol-induced oxidative stress by regulating glutathione metabolism (Harvey et al., 2009; Lu, 2013; Rejitha et al., 2015). Furthermore, the oxidative stress-induced upregulation of Nrf2 is considered to positively modulate expression of VLDLR, which contributes to ALD (Wang et al., 2014b).

In ethanol-exposed mice, the role of Nrf2-induced antioxidant factors was first tested by the Nrf2 inducer D3T (Dong et al., 2008). Upregulation of Nrf2 by D3T treatment has significantly decreased generation of ethanol-induced ROS and apoptosis, which indicated that the activation of Nrf2 could diminish ethanol-induced apoptosis and ameliorate the disease status.

${ }^{1}$ clinicaltrials.gov 


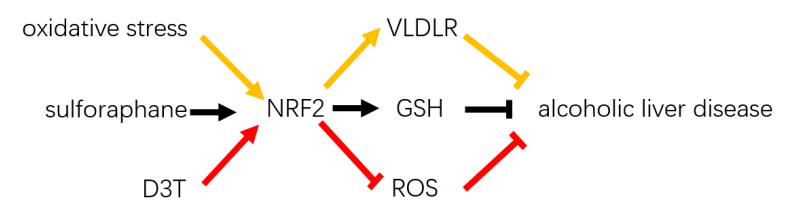

FIGURE 2 | Role of Nrf2 in alcoholic liver disease. Studies shown that oxidative stress promotes Nrf2 activation-induced hepatic VLDLR overexpression and ameliorates ALD. Nrf2 activator-Sulforaphane protects alcoholic fatty liver disease by activating Nrf2 signaling pathway and GSH levels. D3T through upregulate Nrf2 protein levels and decreased ROS levels and liver injuries.

Moreover, Zhou et al. (2014) verified that Nrf2-mediated cytoprotective enzymes could ameliorate alcohol-induced liver steatosis both in in vivo and in vitro models. They further administered sulforaphane, which is an activator of $\mathrm{Nrf} 2$ and present in considerable quantities in brassica vegetables including broccoli, cabbage, and kale, and found it to be effective in improving alcohol-induced liver steatosis (Figure 2). Furthermore, recent advances indicated that activation of the Nrf2 pathway was protective in alcohol-induced liver fibrosis and hepatotoxicity, whereas knockdown of Nrf2 was associated with enhanced alcohol-induced hepatocyte necroptosis (Song et al., 2015; Lu et al., 2016; Ni et al., 2017). By contrast, a more recent study demonstrated that ethyl pyruvate, which has multieffects including antibacterial, anti-inflammatory, antiviral, vasodilatory, antioxidant, and antiapoptotic effects, decreases ALT, AST, hepatic morphological changes, triglycerides, free fatty acids, and the expression of proinflammatory factors and increases the expression of anti-inflammatory factors and peroxisome proliferator-activated receptor- $\alpha$ mRNA which through downregulation of the ROS-Nrf2 signaling pathway, thereby alleviating ALD in mice (Fawcett et al., 1999; Harding et al., 2000; Shen F. et al., 2018). Taken together, these evidences showed that Nrf2 activation plays essential protective role in the development of ALD and that simultaneous downregulation of Nrf2 with ROS and VLDLR may also be effective in the amelioration of ALD (Figure 2). Further studies are required to demonstrate the extent of amelioration between upregulation and downregulation of Nrf2 when ROS and VLDLR expression levels are downregulated in ALD.

\section{PROTECTIVE EFFECTS OF Nrf2 ON VIRAL HEPATITIS-INFECTED CELLS AGAINST OXIDATIVE DAMAGE}

Oxidative stress has been shown to be implicated in viral hepatitis-associated liver diseases, including $\mathrm{HBV}$ and $\mathrm{HCV}$ infections (Bolukbas et al., 2005; Ivanov et al., 2013). A previous study indicated that HCV could mediate the phosphorylation and activation of Nrf2, which was regulated by the mitogen-activated protein kinases. The authors further suggested that the activation of Nrf2-derived survival of HCV-infected cells may provide favorable circumstances for carcinogenesis (Burdette et al.,
2010). Another study showed that the inhibition of Nrf2 and antioxidant response elements is regulated by the core proteins of HCV-replicating cell-triggered delocalization of small Maf proteins, which were bound to NS proteins NS3, thus reducing the expression of cytoprotective genes (Carvajal-Yepes et al., 2011). From the authors' point of view, inhibition of Nrf2 and antioxidant response element-regulated genes may contribute to $\mathrm{HCV}$-associated pathogenesis due to impaired induction of reactive oxygen intermediates caused by cytoprotective genes, which giving rise to host cell DNA damage and promoting the genetic variability of the viral genome. Moreover, Ivanov et al. (2011) found that the antioxidant-protective Nrf2/antioxidant response element pathway is activated by $\mathrm{HCV}$ proteins, including core, E1, E2, NS5A, and NS4B, in an ROS-dependent and -independent manners (Figure 3). In addition, a strong upregulation of the antioxidant-protective system was modulated in the earliest stage, indicating that $\mathrm{Nrf} 2$ is activated to protect against $\mathrm{HCV}$-induced oxidative stress in the acute stage of $\mathrm{HCV}$ infection. In addition, replication of $\mathrm{HCV}$ has been reported to be suppressed by Nrf2-mediated heme oxygenase-1 (HO1) inducible factor, which is a phytocompound isolated from Lindera erythrocarpa Makino fruits (lucidone), and a quinone methide triterpene isolated from Tripterygium wilfordii root extract (celastrol) (Chen et al., 2013; Tseng et al., 2017). Furthermore, an in vitro cell line study from Japan found that knockdown of Nrf2 significantly reduced HCV infection and steatosis (Sugiyama et al., 2014). Most recently, the authors further confirmed that an Nrf2 inhibitor (brusatol) had anti-HCV effects in vitro (Murakami et al., 2018) (Figure 3).

Hepatitis B virus infection, which causes acute or chronic liver inflammation and contributes to the development of HCC, has been shown to induce activation of Nrf2 and antioxidative response elements in vivo and in vitro by HBV-regulatory proteins, including $\mathrm{HBx}$ and large surface proteins, via c-Raf and mitogen-activated protein kinase (Hildt et al., 2002; Schaedler et al., 2010). In addition, the HBx protein-mediated activation of Nrf2 has been introduced to trigger the upregulation of glucose-6-phosphate dehydrogenase, thereby reprogramming metabolism of glucose, and may participate in the development of HCC (Liu et al., 2015). Therefore, Nrf2 is not only a crucial factor that is activated to defend against viral hepatitis-induced

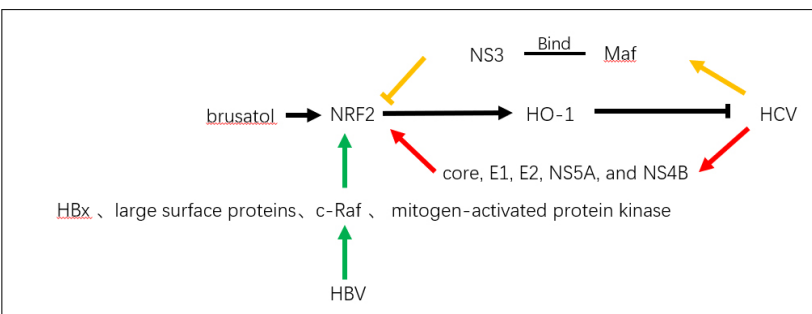

FIGURE 3 | Role of Nrf2 in viral hepatitis-associated liver diseases. Hepatitis $\mathrm{C}$ virus was reported that impaired the protection of $\mathrm{Nrf2}$ by promoting sMaf bound to NS3 and brusatol suppressed HCV infection by promoting Nrf2 pathway. HBV-regulatory proteins, including its $X$ protein $(\mathrm{HBX})$ and large surface proteins were reported to activate Nrf2 and antioxidative responses via c-Raf and mitogen-activated protein kinase. 
oxidative stress but also a protective factor that is involved in the survival of viral hepatitis-infected cells and may contribute to hepatocarcinogenesis.

\section{PROTECTIVE IMPACT OF Nrf2 IN NON-ALCOHOLIC FATTY LIVER DISEASE}

Non-alcoholic fatty liver disease is a progressive disease arising from the accumulation of lipids in hepatocytes and has an increasing incidence worldwide (Satapathy and Sanyal, 2015). Approximately one-third of patients with NAFLD progress to severe $\mathrm{NASH}$, which is linked with inflammation and cirrhosis (Tarantino and Finelli, 2013; Dietrich and Hellerbrand, 2014). Recent studies indicated that ROS and electrophiles are associated with the pathogenesis of NASH; thus, induction of Nrf2 seemed to be promising in the prevention and treatment of NAFLD (Chambel et al., 2015). Du et al. (2016), explored the therapeutic impact of Nrf2 activation by using osteocalcin, and found that it could improve NAFLD by ameliorating oxidative stress and inhibiting the JNK pathway, which is an important pathway involved in the pathogenesis of NAFLD. A recent study demonstrated that scutellarin, a flavonoid glycoside that has an antioxidative stress effect, significantly reduced blood lipid levels and enhanced the antioxidative capacity by activating PPAR $\gamma$ and its coactivator- $1 \alpha, \mathrm{Nrf} 2, \mathrm{HO}-1, \mathrm{GST}$, and NQO1, and suppressing nuclear factor $\kappa \mathrm{B}$ and Keap 1 at the mRNA and protein levels, thus ameliorating NAFLD (Zhang et al., 2018) (Figure 4). In addition, a modulator of PPAR $\gamma$, apigenin, was also revealed to attenuate NAFLD by Nrf2-associated regulation of oxidative stress and hepatocyte lipid metabolism (Feng et al., 2017). Moreover, for the prevention of NAFLD, scutellarin, which is a natural drug with active components of breviscapine, was shown to be effective by enhancing the Nrf2-mediated antioxidant system in high-fat dietand chronic stress-subjected rats (Fan et al., 2017).

Nuclear erythroid 2-related factor 2 has been found to be a key regulator in the protection against NASH (Gupte et al., 2013). By contrast, loss of Nrf2 or deletion of Nrf2 has been found to cause benign steatosis to develop into NASH and contribute to exacerbation of disease status (Chowdhry et al., 2010; Wang et al., 2013). Ramadori et al. (2017) indicated that overactivation of Nrf2 suppressed the hepatocyte-specific c-met deletion (an accelerative factor for NASH)-induced deleterious impact on the progression of $\mathrm{NASH}$ and suggested that $\mathrm{Nrf} 2$ repaired liver damage in hepatocyte-specific c-met-deficient mice via

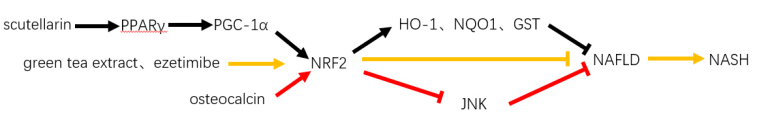

FIGURE 4 | Role of Nrf2 in NAFLD. Multiple studies have shown that several pathways could activate Nrf2 and inhibit NAFLD and NASH including scutellarin-PPAR $\gamma / \mathrm{PGC} 1 \alpha-\mathrm{Nrf2}-\mathrm{HO}-1 / \mathrm{NQO1/GST}$ pathway; green tea extract and ezetimibe activated Nrf2 pathway; osteocalcin activated Nrf2 and subsequently inhibited JNK pathway. maintaining balance in cellular redox homeostasis. To date, green tea extract and ezetimibe (an inhibitor of Niemann-Pick-C1-Like 1) have been revealed to promote the protective impact of Nrf2 against lipid accumulation and the inflammatory response during NASH (Lee et al., 2016; Li et al., 2016) (Figure 4). However, Nrf2-associated therapeutic approaches for NASH remain to be implemented in a real-world clinical manner in the near future.

\section{Nrf2 IN PRIMARY LIVER CANCER}

Hepatocellular carcinoma is the most common primary liver cancer, accounting for more than $80 \%$ of all hepatic malignancies (Forner et al., 2018), with molecular alterations in HCC arising in the very early stage of carcinogenesis (Pitot, 2007). Among the changes, activation of Nrf2 was found to be the prominent pathway that contributes to the progression of preneoplastic lesion to malignancy, which was confirmed by in vivo detection of the inhibition of the Nrf2 pathway that accompanied the regression of cytokeratin 19-positive nodules (Petrelli et al., 2014). The persistent activation of this transcription factor was found to be associated with the accumulation of p62, thus participating in the development of HCC (Inami et al., 2011). This finding was further supported by Saito et al. (2016) who confirmed the promotive impact of p62 in HCV-positive HCC through Nrf2-dependent metabolic reprogramming. In addition, Nrf2 was also found to participate in protection of HCC cells by facilitating the survival response of FGF19 to endoplasmic reticulum stress (Teng et al., 2017; Tian et al., 2018) (Figure 5). Thus, advances were made to regulate the Nrf2 pathway in HCC, including identification of miR-340, miR-144, camptothecin, and valproic acid, which were revealed to be effective in suppressing the Nrf2-dependent pathway, thereby sensitizing HCC cells to anticancer treatments (Shi et al., 2014; Zhou et al., 2016; Chen et al., 2017; Yu et al., 2017) (Figure 5). Moreover, indazolo[3,2-b]quinazolinones were revealed to attack HCC cells by suppressing Nrf2/antioxidative response elements and inducing mitochondrial-dependent apoptosis simultaneously (Zhang et al., 2016). In a clinical retrospective study, patients with high expression levels of Nrf2 $(n=48)$ had significantly reduced overall (median, 13.87 months) and disease-free survival (median, 11.24 months) compared with patients with low expression levels of $\operatorname{Nrf2}(n=17)$, who exhibited median overall survival of 30.40 months and

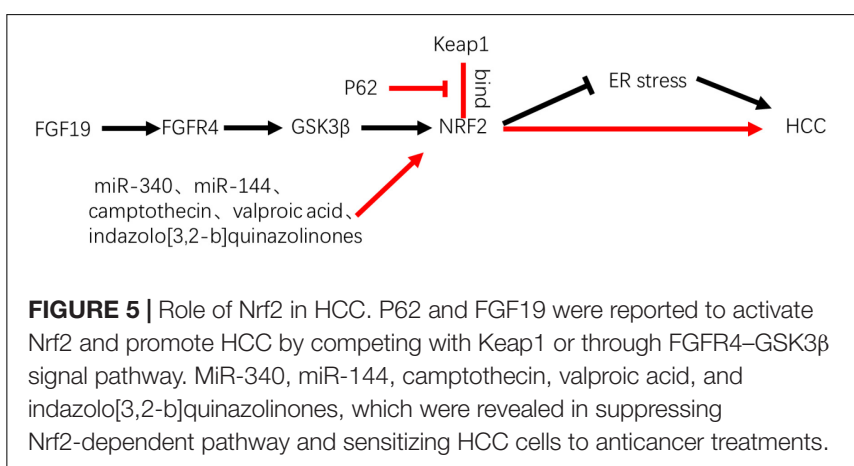


disease-free survival of 24.43 months ( $\mathrm{P}<0.01$ ) (Zhang et al., 2015). The relative risk of high Nrf2 levels in overall survival was 5.96 with 95\% confidence interval of $2.46-14.69(P<0.01)$. However, regarding the sample size and retrospective nature, a large-sized prospective clinical study is required to confirm the prognostic impact of Nrf2 in patients with HCC.

\section{CONCLUSION}

In this review, we briefly summarized the biology characteristics of Nrf2 pathway and discussed the potential therapeutic applications of targeting Nrf2 in liver diseases. To date, there are currently few pharmacological options available to prevent or treat liver diseases. Recently, in clinical trial, NGM282, an engineered FGF19 analog, could significantly reduce liver fat content in patients with NASH and remarkably improve ALP and transaminase levels in patients with PBC (Harrison et al., 2018; Mayo et al., 2018). The small molecule PRI-724 also identified the anti-fibrotic effects in a phase 1 trial in patients with $\mathrm{HCV}$ cirrhosis (Kudo et al., 2018).

A link between liver diseases and oxidative stress is indispensable. The Nrf2 antioxidant pathway is activated to protect the liver by modulating defensive genes, which even

\section{REFERENCES}

Alam, J., Stewart, D., Touchard, C., Boinapally, S., Choi, A. M., and Cook, J. L. (1999). Nrf2, a Cap'n'Collar transcription factor, regulates induction of the heme oxygenase-1 gene. J. Biol. Chem. 274, 26071-26078. doi: 10.1074/jbc.274. 37.26071

Bae, S. H., Sung, S. H., Cho, E. J., Lee, S. K., Lee, H. E., Woo, H. A., et al. (2011). Concerted action of sulfiredoxin and peroxiredoxin I protects against alcohol-induced oxidative injury in mouse liver. Hepatology (Baltimore, $\mathrm{Md}$ ) 53, 945-953. doi: 10.1002/hep.24104

Bataille, A. M., and Manautou, J. E. (2012). Nrf2: a potential target for new therapeutics in liver disease. Clin. Pharmacol. Ther. 92, 340-348. doi: 10.1038/ clpt.2012.110

Bellezza, I., Giambanco, I., Minelli, A., and Donato, R. (2018). Nrf2-Keap1 signaling in oxidative and reductive stress. Biochim. Biophys. Acta 1865, 721-733. doi: 10.1016/j.bbamcr.2018.02.010

Bolukbas, C., Bolukbas, F. F., Horoz, M., Aslan, M., Celik, H., and Erel, O. (2005). Increased oxidative stress associated with the severity of the liver disease in various forms of hepatitis B virus infection. BMC Infect Dis. 5:95. doi: 10.1186/ 1471-2334-5-95

Burdette, D., Olivarez, M., and Waris, G. (2010). Activation of transcription factor Nrf2 by hepatitis $\mathrm{C}$ virus induces the cell-survival pathway. J. Gen. Virol. 91, 681-690. doi: 10.1099/vir.0.014340-0

Cao, M., Wang, H., Guo, L., Yang, S., Liu, C., Khor, T. O., et al. (2017). Dibenzoylmethane protects against $\mathrm{CCl} 4$-induced acute liver injury by activating Nrf2 via JNK, AMPK, and calcium signaling. AAPS J. 19, 1703-1714. doi: 10.1208/s12248-017-0133-1

Carvajal-Yepes, M., Himmelsbach, K., Schaedler, S., Ploen, D., Krause, J., Ludwig, L., et al. (2011). Hepatitis C virus impairs the induction of cytoprotective Nrf2 target genes by delocalization of small Maf proteins. J. Biol. Chem. 286, 8941-8951. doi: 10.1074/jbc.M110. 186684

Chambel, S. S., Santos-Goncalves, A., and Duarte, T. L. (2015). The dual role of $\mathrm{Nrf2}$ in nonalcoholic fatty liver disease: regulation of antioxidant defenses and hepatic lipid metabolism. Biomed. Res. Int. 2015:597134. doi: 10.1155/2015/ 597134

Chen, F., Wang, H., Zhu, J., Zhao, R., Xue, P., Zhang, Q., et al. (2017). Camptothecin suppresses NRF2-ARE activity and sensitises hepatocellular protect viral hepatitis-infected cells and HCC cells. A number of preclinical studies have detected regulatory factors for Nrf2; however, further identification of Nrf2 activators for liver injury/failure and Nrf2 inhibitors for viral hepatitis, and HCC is promising for the establishment of extensive and effective approaches to improve the prognosis of liver diseases. Regarding the great potential of this transcription factor, there is an unmet need for prospective clinical trials to explore the therapeutic impact of Nrf2 regulation in patients with liver diseases.

\section{AUTHOR CONTRIBUTIONS}

DX and MX wrote the manuscript. SJ and YQ wrote some part of the manuscript and made language retouching for our manuscript. HW revised the manuscript. QX and XK designed and revised the manuscript.

\section{FUNDING}

This work was supported by the National Natural Science Foundation of China (81670562 to XK and 81670598 to QX).

carcinoma cells to anticancer drugs. Br. J. Cancer 117, 1495-1506. doi: 10.1038/ bjc. 2017.317

Chen, W. C., Wang, S. Y., Chiu, C. C., Tseng, C. K., Lin, C. K., Wang, H. C., et al. (2013). Lucidone suppresses hepatitis C virus replication by Nrf2-mediated heme oxygenase-1 induction. Antimicrob. Agents Chemother. 57, 1180-1191. doi: 10.1128/AAC.02053-12

Chowdhry, S., Nazmy, M. H., Meakin, P. J., Dinkova-Kostova, A. T., Walsh, S. V., Tsujita, T., et al. (2010). Loss of Nrf2 markedly exacerbates nonalcoholic steatohepatitis. Free Radic. Biol. Med. 48, 357-371. doi: 10.1016/j. freeradbiomed.2009.11.007

Dey, A., and Cederbaum, A. I. (2006). Alcohol and oxidative liver injury. Hepatology (Baltimore, Md) 43, S63-S74. doi: 10.1002/hep.20957

Dietrich, P., and Hellerbrand, C. (2014). Non-alcoholic fatty liver disease, obesity and the metabolic syndrome. Best Pract. Res. Clin. Gastroenterol. 28, 637-653. doi: 10.1016/j.bpg.2014.07.008

Dong, J., Sulik, K. K., and Chen, S. Y. (2008). Nrf2-mediated transcriptional induction of antioxidant response in mouse embryos exposed to ethanol in vivo: implications for the prevention of fetal alcohol spectrum disorders. Antioxid. Redox Signal. 10, 2023-2033. doi: 10.1089/ars.2007.2019

Du, J., Zhang, M., Lu, J., Zhang, X., Xiong, Q., Xu, Y., et al. (2016). Osteocalcin improves nonalcoholic fatty liver disease in mice through activation of Nrf2 and inhibition of JNK. Endocrine 53, 701-709. doi: 10.1007/s12020-016-0926-5

Fan, H., Ma, X., Lin, P., Kang, Q., Zhao, Z., Wang, L., et al. (2017). Scutellarin prevents nonalcoholic fatty liver disease (NAFLD) and hyperlipidemia via PI3K/AKT-dependent activation of nuclear factor (erythroid-derived 2)-like 2 (nrf2) in Rats. Med. Sci. Monit. 23, 5599-5612. doi: 10.12659/MSM.90 7530

Fawcett, T. W., Martindale, J. L., Guyton, K. Z., Hai, T., and Holbrook, N. J. (1999). Complexes containing activating transcription factor (ATF)/cAMP-responsiveelement-binding protein (CREB) interact with the CCAAT/enhancer-binding protein (C/EBP)-ATF composite site to regulate Gadd153 expression during the stress response. Biochem. J. 339(Pt 1), 135-141.

Feng, X., Yu, W., Li, X., Zhou, F., Zhang, W., Shen, Q., et al. (2017). Apigenin, a modulator of PPARgamma, attenuates HFD-induced NAFLD by regulating hepatocyte lipid metabolism and oxidative stress via Nrf2 activation. Biochem. Pharmacol. 136, 136-149. doi: 10.1016/j.bcp.2017.04.014

Forner, A., Reig, M., and Bruix, J. (2018). Hepatocellular carcinoma. Lancet 391, 1301-1314. doi: 10.1016/S0140-6736(18)30010-2 
Ge, M., Yao, W., Yuan, D., Zhou, S., Chen, X., Zhang, Y., et al. (2017). Brg1-mediated Nrf2/HO-1 pathway activation alleviates hepatic ischemiareperfusion injury. Cell Death Dis. 8:e2841. doi: 10.1038/cddis.2017.236

Gupte, A. A., Lyon, C. J., and Hsueh, W. A. (2013). Nuclear factor (erythroidderived 2)-like-2 factor (Nrf2), a key regulator of the antioxidant response to protect against atherosclerosis and nonalcoholic steatohepatitis. Curr. Diabetes Rep. 13, 362-371. doi: 10.1007/s11892-013-0372-1

Harding, H. P., Zhang, Y., Bertolotti, A., Zeng, H., and Ron, D. (2000). Perk is essential for translational regulation and cell survival during the unfolded protein response. Mol. Cell 5, 897-904. doi: 10.1016/S1097-2765(00)80330-5

Harrison, S. A., Rinella, M. E., Abdelmalek, M. F., Trotter, J. F., Paredes, A. H., Arnold, H. L., et al. (2018). NGM282 for treatment of non-alcoholic steatohepatitis: a multicentre, randomised, double-blind, placebo-controlled, phase 2 trial. Lancet 391, 1174-1185. doi: 10.1016/S0140-6736(18)30474-4

Harvey, C. J., Thimmulappa, R. K., Singh, A., Blake, D. J., Ling, G., Wakabayashi, N., et al. (2009). Nrf2-regulated glutathione recycling independent of biosynthesis is critical for cell survival during oxidative stress. Free Radic. Biol. Med. 46, 443-453. doi: 10.1016/j.freeradbiomed.2008.10.040

Hennig, P., Garstkiewicz, M., and Grossi, S. (2018). The Crosstalk between Nrf2 and Inflammasomes. Int. J. Mol. Sci. 19:E562. doi: 10.3390/ijms190 20562

Hildt, E., Munz, B., Saher, G., Reifenberg, K., and Hofschneider, P. H. (2002). The PreS2 activator MHBs(t) of hepatitis B virus activates $c$-raf-1/Erk2 signaling in transgenic mice. EMBO J. 21, 525-535. doi: 10.1093/emboj/21. 4.525

Huang, Y. J., Chen, P., Lee, C. Y., Yang, S. Y., Lin, M. T., Lee, H. S., et al. (2016). Protection against acetaminophen-induced acute liver failure by omentum adipose tissue derived stem cells through the mediation of Nrf2 and cytochrome P450 expression. J. Biomed. Sci. 23:5. doi: 10.1186/s12929-016-0231-x

Inami, Y., Waguri, S., Sakamoto, A., Kouno, T., Nakada, K., Hino, O., et al. (2011). Persistent activation of Nrf2 through p62 in hepatocellular carcinoma cells. J. Cell Biol. 193, 275-284. doi: 10.1083/jcb.201102031

Ivanov, A. V., Bartosch, B., Smirnova, O. A., Isaguliants, M. G., and Kochetkov, S. N. (2013). HCV and oxidative stress in the liver. Viruses 5, 439-469. doi: $10.3390 / \mathrm{v} 5020439$

Ivanov, A. V., Smirnova, O. A., Ivanova, O. N., Masalova, O. V., Kochetkov, S. N., and Isaguliants, M. G. (2011). Hepatitis C virus proteins activate NRF2/ARE pathway by distinct ROS-dependent and independent mechanisms in HUH7 cells. PLoS One 6:e24957. doi: 10.1371/journal.pone.0024957

Karin, M., and Dhar, D. (2016). Liver carcinogenesis: from naughty chemicals to soothing fat and the surprising role of NRF2. Carcinogenesis 37, 541-546. doi: 10.1093/carcin/bgw060

Ke, B., Shen, X. D., Zhang, Y., Ji, H., Gao, F., Yue, S., et al. (2013). KEAP1-NRF2 complex in ischemia-induced hepatocellular damage of mouse liver transplants. J. Hepatol. 59, 1200-1207. doi: 10.1016/j.jhep.2013.07.016

Klaassen, C. D., and Reisman, S. A. (2010). Nrf2 the rescue: effects of the antioxidative/electrophilic response on the liver. Toxicol. Appl. Pharmacol. 244, 57-65. doi: 10.1016/j.taap.2010.01.013

Kudo, M., Finn, R. S., Qin, S., Han, K. H., Ikeda, K., Piscaglia, F., et al. (2018). Lenvatinib versus sorafenib in first-line treatment of patients with unresectable hepatocellular carcinoma: a randomised phase 3 non-inferiority trial. Lancet 391, 1163-1173. doi: 10.1016/S0140-6736(18)30207-1

Kudoh, K., Uchinami, H., Yoshioka, M., Seki, E., and Yamamoto, Y. (2014). Nrf2 activation protects the liver from ischemia/reperfusion injury in mice. Ann. Surg. 260, 118-127. doi: 10.1097/SLA.0000000000000287

Lee, D. H., Han, D. H., Nam, K. T., Park, J. S., Kim, S. H., Lee, M., et al. (2016). Ezetimibe, an NPC1L1 inhibitor, is a potent Nrf2 activator that protects mice from diet-induced nonalcoholic steatohepatitis. Free Radic. Biol. Med. 99, 520-532. doi: 10.1016/j.freeradbiomed.2016.09.009

Li, J., Sapper, T. N., Mah, E., Rudraiah, S., Schill, K. E., Chitchumroonchokchai, C., et al. (2016). Green tea extract provides extensive Nrf2-independent protection against lipid accumulation and NFkappaB pro- inflammatory responses during nonalcoholic steatohepatitis in mice fed a high-fat diet. Mol. Nutr. Food Res. 60, 858-870. doi: 10.1002/mnfr.201500814

Liu, B., Fang, M., He, Z., Cui, D., Jia, S., Lin, X., et al. (2015). Hepatitis B virus stimulates G6PD expression through HBx-mediated Nrf2 activation. Cell Death Dis. 6, e1980. doi: 10.1038/cddis.2015.322
Liu, M., Reddy, N. M., Higbee, E. M., Potteti, H. R., Noel, S., Racusen, L., et al. (2014). The Nrf2 triterpenoid activator, CDDO-imidazolide, protects kidneys from ischemia-reperfusion injury in mice. Kid. Int. 85, 134-141. doi: 10.1038/ ki.2013.357

Liu, X., Wang, T., Liu, X., Cai, L., Qi, J., Zhang, P., et al. (2016). Biochanin A protects lipopolysaccharide/D-galactosamine-induced acute liver injury in mice by activating the Nrf2 pathway and inhibiting NLRP3 inflammasome activation. Int. Immunopharmacol. 38, 324-331. doi: 10.1016/j.intimp.2016.0 6.009

Lu, C., Xu, W., Zhang, F., Shao, J., and Zheng, S. (2016). Nrf2 knockdown disrupts the protective effect of curcumin on alcohol-induced hepatocyte necroptosis. Mol. Pharm. 13, 4043-4053. doi: 10.1021/acs.molpharmaceut.6b 00562

Lu, S. C. (2013). Glutathione synthesis. Biochim. Biophys. Acta 2013, 3143-3153. doi: 10.1016/j.bbagen.2012.09.008

Mayo, M. J., Wigg, A. J., Leggett, B. A., Arnold, H., Thompson, A. J., Weltman, M., et al. (2018). NGM282 for treatment of patients with primary biliary cholangitis: a multicenter, randomized, double-blind, placebo-controlled trial. Hepatol. Commun. 2, 1037-1050. doi: 10.1002/hep4.1209

Murakami, Y., Sugiyama, K., Ebinuma, H., Nakamoto, N., Ojiro, K., Chu, P. S., et al. (2018). Dual effects of the Nrf2 inhibitor for inhibition of hepatitis $C$ virus and hepatic cancer cells. BMC Cancer 18:680. doi: 10.1186/s12885-018-4588-y

Ni, Y. H., Huo, L. J., and Li, T. T. (2017). Antioxidant axis Nrf2-keap1-ARE in inhibition of alcoholic liver fibrosis by IL-22. World J. Gastroenterol. 23, 2002-2011. doi: 10.3748/wjg.v23.i11.2002

Pan, C. W., Pan, Z. Z., Hu, J. J., Chen, W. L., Zhou, G. Y., Lin, W., et al. (2016). Mangiferin alleviates lipopolysaccharide and D-galactosamine-induced acute liver injury by activating the Nrf2 pathway and inhibiting NLRP3 inflammasome activation. Eur. J. Pharmacol. 770, 85-91. doi: 10.1016/j.ejphar. 2015.12.006

Pan, C. W., Yang, S. X., Pan, Z. Z., Zheng, B., Wang, J. Z., Lu, G. R., et al. (2017). Andrographolide ameliorates d-galactosamine/lipopolysaccharideinduced acute liver injury by activating Nrf2 signaling pathway. Oncotarget 8 , 41202-41210. doi: 10.18632/oncotarget.17149

Peng, X., Dai, C., Liu, Q., Li, J., and Qiu, J. (2018). Curcumin attenuates on carbon tetrachloride-induced acute liver injury in mice via modulation of the Nrf2/HO-1 and TGF-beta1/Smad3 pathway. Molecules (Basel, Switzerland) 23:E215. doi: 10.3390/molecules23010215

Petrelli, A., Perra, A., Cora, D., Sulas, P., Menegon, S., Manca, C., et al. (2014). MicroRNA/gene profiling unveils early molecular changes and nuclear factor erythroid related factor 2 (NRF2) activation in a rat model recapitulating human hepatocellular carcinoma (HCC). Hepatology (Baltimore, Md) 59, 228-241. doi: 10.1002/hep.26616

Pitot, H. C. (2007). Adventures in hepatocarcinogenesis. Annu. Rev. Pathol. 2, 1-29. doi: 10.1146/annurev.pathol.2.010506.092027

Ramadori, P., Drescher, H., Erschfeld, S., Fragoulis, A., Kensler, T. W., Wruck, C. J., et al. (2017). Genetic Nrf2 overactivation inhibits the deleterious effects induced by hepatocyte-specific c-met deletion during the progression of NASH. Oxid. Med. Cell. Longev. 2017:3420286. doi: 10.1155/2017/3420286

Rao, J., Qian, X., Li, G., Pan, X., Zhang, C., Zhang, F., et al. (2015). ATF3mediated NRF2/HO-1 signaling regulates TLR4 innate immune responses in mouse liver ischemia/reperfusion injury. Am. J. Transplant 15, 76-87. doi: $10.1111 /$ ajt.12954

Reddy, N. M., Suryanaraya, V., Yates, M. S., Kleeberger, S. R., Hassoun, P. M., Yamamoto, M., et al. (2009). The triterpenoid CDDO-imidazolide confers potent protection against hyperoxic acute lung injury in mice. Am. J. Respir. Crit. Care Med. 180, 867-874. doi: 10.1164/rccm.200905-06700C

Rejitha, S., Prathibha, P., and Indira, M. (2015). Nrf2-mediated antioxidant response by ethanolic extract of Sida cordifolia provides protection against alcohol-induced oxidative stress in liver by upregulation of glutathione metabolism. Redox Rep. 20, 75-80. doi: 10.1179/1351000214Y.0000000108

Rojo de la Vega, M., Chapman, E., and Zhang, D. D. (2018). NRF2 and the hallmarks of cancer. Cancer Cell 34, 21-43. doi: 10.1016/j.ccell.2018.03.022

Saito, T., Ichimura, Y., Taguchi, K., Suzuki, T., Mizushima, T., Takagi, K., et al. (2016). p62/Sqstm1 promotes malignancy of HCV-positive hepatocellular carcinoma through Nrf2-dependent metabolic reprogramming. Nat Commun. 7:12030. doi: $10.1038 /$ ncomms 12030 
Satapathy, S. K., and Sanyal, A. J. (2015). Epidemiology and natural history of nonalcoholic fatty liver disease. Semin. Liver Dis. 35, 221-235. doi: 10.1055/s0035-1562943

Schaedler, S., Krause, J., Himmelsbach, K., Carvajal-Yepes, M., Lieder, F., Klingel, K., et al. (2010). Hepatitis B virus induces expression of antioxidant response element-regulated genes by activation of Nrf2. J. Biol. Chem. 285, 41074-41086. doi: 10.1074/jbc.M110.145862

Shen, F., Wang, Z., Liu, W., and Liang, Y. (2018a). Ethyl pyruvate can alleviate alcoholic liver disease through inhibiting Nrf2 signaling pathway. Exp. Ther Med. 15, 4223-4228. doi: 10.3892/etm.2018.5925

Shen, Z., Wang, Y., Su, Z., Kou, R., Xie, K., and Song, F. (2018b). Activation of p62keap1-Nrf2 antioxidant pathway in the early stage of acetaminophen-induced acute liver injury in mice. Chem. Biol. Interact. 282, 22-28. doi: 10.1016/j.cbi. 2018.01.008

Shepard, B. D., Tuma, D. J., and Tuma, P. L. (2010). Chronic ethanol consumption induces global hepatic protein hyperacetylation. Alcohol. Clin. Exp. Res. 34, 280-291. doi: 10.1111/j.1530-0277.2009.01091.x

Shi, L., Chen, Z. G., Wu, L. L., Zheng, J. J., Yang, J. R., Chen, X. F., et al. (2014). miR-340 reverses cisplatin resistance of hepatocellular carcinoma cell lines by targeting Nrf2-dependent antioxidant pathway. Asian Pac J. Cancer Prev. 15, 10439-10444. doi: 10.7314/APJCP.2014.15.23.10439

Song, X., Yin, S., Huo, Y., Liang, M., Fan, L., Ye, M., et al. (2015) Glycycoumarin ameliorates alcohol-induced hepatotoxicity via activation of Nrf2 and autophagy. Free Radic. Biol. Med. 89, 135-146. doi: 10.1016/j. freeradbiomed.2015.07.006

Sugiyama, K., Ebinuma, H., Nakamoto, N., Sakasegawa, N., Murakami, Y., Chu, P. S., et al. (2014). Prominent steatosis with hypermetabolism of the cell line permissive for years of infection with hepatitis C virus. PLoS One 9:e94460. doi: 10.1371/journal.pone.0094460

Sussan, T. E., Rangasamy, T., Blake, D. J., Malhotra, D., El-Haddad, H., Bedja, D., et al. (2009). Targeting Nrf2 with the triterpenoid CDDO-imidazolide attenuates cigarette smoke-induced emphysema and cardiac dysfunction in mice. Proc. Natl. Acad. Sci. U.S.A. 106, 250-255. doi: 10.1073/pnas.08043 33106

Tang, W., Jiang, Y. F., Ponnusamy, M., and Diallo, M. (2014). Role of Nrf2 in chronic liver disease. World J. Gastroenterol. 20, 13079-13087. doi: 10.3748/wjg. v20.i36.13079

Tarantino, G., and Finelli, C. (2013). Pathogenesis of hepatic steatosis: the link between hypercortisolism and non-alcoholic fatty liver disease. World $J$ Gastroenterol. 19, 6735-6743. doi: 10.3748/wjg.v19.i40.6735

Teng, Y., Zhao, H., Gao, L., Zhang, W., Shull, A. Y., and Shay, C. (2017). FGF19 protects hepatocellular carcinoma cells against endoplasmic reticulum stress via activation of FGFR4-GSK3beta-Nrf2 signaling. Cancer Res. 77, 6215-6225. doi: 10.1158/0008-5472.CAN-17-2039

Thimmulappa, R. K., Fuchs, R. J., Malhotra, D., Scollick, C., Traore, K., Bream, J. H., et al. (2007). Preclinical evaluation of targeting the Nrf2 pathway by triterpenoids (CDDO-Im and CDDO-Me) for protection from LPS-induced inflammatory response and reactive oxygen species in human peripheral blood mononuclear cells and neutrophils. Antioxid. Redox Signal. 9, 1963-1970. doi: 10.1089 /ars.2007.1745

Tian, B., Lu, Z. N., and Guo, X. L. (2018). Regulation and role of nuclear factorE2-related factor 2 (Nrf2) in multidrug resistance of hepatocellular carcinoma. Chem. Biol. Interact. 280, 70-76. doi: 10.1016/j.cbi.2017.12.014

Tian, Y., Li, Z., Shen, B., Zhang, Q., and Feng, H. (2017). Protective effects of morin on lipopolysaccharide/d-galactosamine-induced acute liver injury by inhibiting TLR4/NF-kappaB and activating Nrf2/HO-1 signaling pathways. Int. Immunopharmacol. 45, 148-155. doi: 10.1016/j.intimp.2017.02.010

Tseng, C. K., Hsu, S. P., Lin, C. K., Wu, Y. H., Lee, J. C., and Young, K. C. (2017) Celastrol inhibits hepatitis $\mathrm{C}$ virus replication by upregulating heme oxygenase1 via the JNK MAPK/Nrf2 pathway in human hepatoma cells. Antiv. Res. 146, 191-200. doi: 10.1016/j.antiviral.2017.09.010

Vomund, S., Schafer, A., Parnham, M. J., Brüne, B., and von Knethen, A. (2017) Nrf2, the master regulator of anti-oxidative responses. Int. J. Mol. Sci. 18:E2772. doi: 10.3390/ijms18122772
Wang, C., Cui, Y., Li, C., Zhang, Y., Xu, S., Li, X., et al. (2013). Nrf2 deletion causes "benign" simple steatosis to develop into nonalcoholic steatohepatitis in mice fed a high-fat diet. Lipids Health Dis. 12:165. doi: 10.1186/1476-511X-12-165

Wang, W., Wu, L., Li, Q., Zhang, Z., Xu, L., Lin, C., et al. (2018). Madecassoside prevents acute liver failure in LPS/D-GalN-induced mice by inhibiting p38/NFkappaB and activating Nrf2/HO-1 signaling. Biomed. Pharmacother. 103, 1137-1145. doi: 10.1016/j.biopha.2018.04.162

Wang, Y., Kou, Y., Wang, X., Cederbaum, A., and Wang, R. (2014a). Multifactorial comparative proteomic study of cytochrome P450 2E1 function in chronic alcohol administration. PLoS One 9:e92504. doi: 10.1371/journal.pone.0092504

Wang, Z., Dou, X., Li, S., Zhang, X., Sun, X., Zhou, Z., et al. (2014b). Nuclear factor (erythroid-derived 2)-like 2 activation-induced hepatic very-low-density lipoprotein receptor overexpression in response to oxidative stress contributes to alcoholic liver disease in mice. Hepatology (Baltimore, Md) 59, 1381-1392. doi: $10.1002 /$ hep. 26912

Wu, K. C., Liu, J. J., and Klaassen, C. D. (2012). Nrf2 activation prevents cadmiuminduced acute liver injury. Toxicol. Appl. Pharmacol. 263, 14-20. doi: 10.1016/j. taap.2012.05.017

Xie, Y. L., Chu, J. G., Jian, X. M., Dong, J. Z., Wang, L. P., Li, G. X., et al. (2017). Curcumin attenuates lipopolysaccharide/d-galactosamine-induced acute liver injury by activating Nrf2 nuclear translocation and inhibiting NF-kB activation. Biomed. Pharmacother. 91, 70-77. doi: 10.1016/j.biopha.2017.04.070

Xu, D., Chen, L., Chen, X., Wen, Y., Yu, C., Yao, J., et al. (2017). The triterpenoid CDDO-imidazolide ameliorates mouse liver ischemia-reperfusion injury through activating the Nrf2/HO-1 pathway enhanced autophagy. Cell Death Dis. 8:e2983. doi: 10.1038/cddis.2017.386

Yu, J. I., Choi, C., Shin, S. W., Son, A., Lee, G. H., Kim, S. Y., et al. (2017). Valproic acid sensitizes hepatocellular carcinoma cells to proton therapy by suppressing NRF2 activation. Sci. Rep. 7:14986. doi: 10.1038/s41598-017-15165-3

Zhang, F., Wang, S., Zhang, M., Weng, Z., Li, P., Gan, Y., et al. (2012). Pharmacological induction of heme oxygenase-1 by a triterpenoid protects neurons against ischemic injury. Stroke 43, 1390-1397. doi: 10.1161/ STROKEAHA.111.647420

Zhang, M., Zhang, C., Zhang, L., Yang, Q., Zhou, S., Wen, Q., et al. (2015). Nrf2 is a potential prognostic marker and promotes proliferation and invasion in human hepatocellular carcinoma. BMC Cancer 15:531. doi: 10.1186/s12885-015-1541-1

Zhang, X., Ji, R., Sun, H., Peng, J., Ma, X., Wang, C., et al. (2018). Scutellarin ameliorates nonalcoholic fatty liver disease through the PPARgamma/PGC1alpha-Nrf2 pathway. Free Radic. Res. 52, 198-211. doi: 10.1080/10715762. 2017.1422602

Zhang, Y., Qiao, R., He, D., Zhao, Z., Yang, S., Zou, H., et al. (2016). Indazolo[3,2-b]quinazolinones attack hepatocellular carcinoma Hep3B cells by inducing mitochondrial-dependent apoptosis and inhibition of Nrf2/ARE signaling pathway. Curr. Mol. Med. 16, 820-828. doi: 10.2174/1566524016666161128114444

Zhou, R., Lin, J., and Wu, D. (2014). Sulforaphane induces Nrf2 and protects against CYP2E1-dependent binge alcohol-induced liver steatosis. Biochim. Biophys. Acta 1840, 209-218. doi: 10.1016/j.bbagen.2013.09.018

Zhou, S., Ye, W., Zhang, Y., Yu, D., Shao, Q., Liang, J., et al. (2016). miR-144 reverses chemoresistance of hepatocellular carcinoma cell lines by targeting Nrf2-dependent antioxidant pathway. Am. J. Transl. Res. 8, 2992-3002

Conflict of Interest Statement: The authors declare that the research was conducted in the absence of any commercial or financial relationships that could be construed as a potential conflict of interest.

Copyright (C) $2019 \mathrm{Xu}, \mathrm{Xu}$, Jeong, Qian, Wu, Xia and Kong. This is an open-access article distributed under the terms of the Creative Commons Attribution License (CC BY). The use, distribution or reproduction in other forums is permitted, provided the original author(s) and the copyright owner(s) are credited and that the original publication in this journal is cited, in accordance with accepted academic practice. No use, distribution or reproduction is permitted which does not comply with these terms. 